\title{
Computing eigenvalues of banded symmetric Toeplitz matrices
}

\section{Report}

Author(s):

Arbenz, Peter

Publication date:

1989

Permanent link:

https://doi.org/10.3929/ethz-a-000505287

Rights / license:

In Copyright - Non-Commercial Use Permitted

Originally published in:

ETH, Eidgenössische Technische Hochschule Zürich, Departement Informatik, Institut für Wissenschaftliches Rechnen 104 
Eidgenössische

Technische Hochschule

Zürich
Departement Informatik Institut für Wissenschaftliches Rechnen

Peter Arbenz

\section{Computing Eigenvalues of Banded Symmetric Toeplitz Matrices}

Mai 1989 
Author's adress:

Institut für Wissenschaftliches Rechnen

ETH-Zentrum

CH-8092 Zürich, Switzerland

(C) 1989 Departement Informatik, ETH Zürich 


\section{Abstract}

A recently proposed algorithm for the computation of the eigenvalues of symmetric banded Toeplitz matrices is investigated.

The basic idea of the algorithm is to embed the Toeplitz matrix in a symmetric circulant matrix of higher order. After having computed the spectral decomposition of the circulant matrix which is trivial since the eigenvectors of circulants are known a priori and the eigenvalues can be obtained by Fourier transform - the Toeplitz eigenvalue problem is treated as a restricted eigenvalue problem. Doing this, use can be made of the theory for intermediate problems of Weinstein and Aronszajn and its recent refinements.

Since the main part of the proposed algorithm consists of independent searches for zeros in disjoint real intervals, the algorithm is well suited for parallel computers. We discuss implementations of the algorithm and give some numerical results. The (sequential) complexity of the computation of $s$ eigenvalues of the Toeplitz matrix $T$ is $O\left(\operatorname{sr}\left(n+r^{2}\right)\right)$ where $n$ is the order and $r$ the bandwidth of $T$. 
1. Introduction. In this note we pursue an idea that was presented by Golub and the author in [2] to compute eigenvalues and eigenvectors of a symmetric banded Toeplitz matrix. A symmetric banded Toeplitz matrix with bandwidth $r$ is a matrix of the form

$$
T=\left(\begin{array}{ccccc}
t_{0} & \cdots & t_{r} & & \\
\vdots & \ddots & & \ddots & \\
t_{r} & & \ddots & & t_{r} \\
& \ddots & & \ddots & \vdots \\
& & t_{r} & \cdots & t_{0}
\end{array}\right) \in \mathbb{R}^{n \times n},
$$

i.e. a matrix with elements $t_{i j}=t_{|i-j|}$ where $t=\left(t_{0}, \ldots, t_{n-1}\right)^{*}$ is a vector with vanishing components $t_{r+1}, \ldots, t_{n-1} \cdot{ }^{*}$ denotes Hermitian transposition. $\lambda \in \mathbb{R}$ is called an eigenvalue of $T$ if there is a nonzero vector $x \in \mathbb{R}^{n}$ so that

$$
T x=\lambda x .
$$

Such a vector is called an eigenvector of $T$ corresponding to $\lambda$.

Symmetric banded Toeplitz matrices occur e.g. in eigenvalue problems for finite difference matrices originating from one-dimensional differential equations with constant coefficients. In signal processing it can be of interest to know the smallest eigenvalue of symmetric Toeplitz matrices. This problem has been investigated by Cybenko and van Loan [10] and by Hu and Kung [17]. To its solution these authors propose algorithms which combine inverse vector iteration and Rayleigh quotient iteration, respectively, with fast methods for solving $(T-\lambda I) x=y$. In the first mentioned paper this equation is even a Yule-Walker equation.

Trench [22] as well as Bini and Pan [7] developed algorithms for the computation of eigenvalues of banded Toeplitz matrices. These methods are based on algorithms to compute the determinant $f(\lambda):=\operatorname{det}(T-\lambda I)$ and its derivative $f^{\prime}(\lambda)$ in $O\left(r^{2} \log n+r^{3}\right)$ flops or in $O\left(r \log r \log n+r^{3}\right)$ flops if fast Fourier transform is applicable. 
Let

$$
P:=0_{r} \oplus I_{n}=\operatorname{diag}\left(0_{r}, I_{n}\right) \in \mathbb{R}^{m \times m}, \quad m=n+r,
$$

where $I_{n}$ and $0_{r}$ are the identity and zero matrices of order $n$ and $r$, respectively.

In [2] it was noted that the eigenvalue problem (1.2) is equivalent to

$$
P C P y=\lambda y, \quad P y=y
$$

where

$$
C=\left(\begin{array}{cccccccc}
t_{0} & \cdots & t_{r} & & & t_{r} & \cdots & t_{1} \\
\vdots & \ddots & & \ddots & & & \ddots & \vdots \\
t_{r} & & \ddots & & \ddots & & & t_{r} \\
& \ddots & & \ddots & & \ddots & \\
& & \ddots & & \ddots & & \ddots & \\
t_{r} & & & \ddots & & \ddots & & t_{r} \\
\vdots & \ddots & & & \ddots & & \ddots & \vdots \\
t_{1} & \cdots & t_{r} & & & t_{r} & \cdots & t_{0}
\end{array}\right) \in \mathbb{R}^{m \times m}
$$

is a symmetric circulant matrix. Note that $P C P=0_{r} \oplus T$. (1.4) is a restricted eigenvalue problem as considered in detail in $[1,4]$. The extension of Toeplitz matrices to circulant ones is classic. Its use for banded matrices has been proposed by Jain [18] to solve the linear equation $T x=y$. $C$ was also used by Bini and Capovani [6] to derive some spectral properties of the Toeplitz matrix $T$.

In $\S 2$ we deal with the eigenvalue problem of circulant matrices which can be solved very quickly by the Fourier transform. In $\S 3$ we review the theory of restricted eigenvalue problems of the form (1.4). We will show how the eigenvalues and eigenvectors of a restricted eigenvalue problem can be computed from those of the unconstrained eigenvalue problem $C x=\lambda x$. In $\S 4$ we apply the results obtained in $\S \S 2$ and 3 to problem (1.4)-(1.5) and in the final section 5 we report on numerical experiments. 
2. Circulant matrices. Let

(2.1) $C=\operatorname{circ}(c):=\left(\begin{array}{ccccc}c_{0} & c_{1} & \cdots & c_{m-2} & c_{m-1} \\ c_{m-1} & c_{0} & \cdots & c_{m-3} & c_{m-2} \\ \vdots & \vdots & \ddots & \vdots & \vdots \\ c_{2} & c_{3} & \cdots & c_{0} & c_{1} \\ c_{1} & c_{2} & \cdots & c_{m-1} & c_{0}\end{array}\right) \in \mathbb{R}^{m \times m}$

be a circulant matrix. $c \in \mathbb{R}^{m}$ is the column vector with elements $c_{0}, \ldots, c_{m-1}$. Then

$$
C=\sum_{j=0}^{m-1} c_{j} \Pi^{j}
$$

where $I I$ is the circulant matrix

$$
\Pi=\left(\begin{array}{cccc}
0 & 1 & & \\
& \ddots & \ddots & \\
& & \ddots & 1 \\
1 & & & 0
\end{array}\right)
$$

The spectral decomposition of $\Pi$ is known to be [11, p.72]

$$
\Pi=F^{*} \Omega F,
$$

where

$$
\Omega=\operatorname{diag}\left(1, \omega, \omega^{2}, \ldots, \omega^{m-1}\right), \quad \omega=e^{2 \pi i / m}, \quad i=\sqrt{-1},
$$

and

$$
F=\left(f_{k j}\right):=\frac{1}{\sqrt{m}}\left(\bar{\omega}^{k j}\right), \quad 0 \leq k, j \leq m-1 .
$$

$\bar{\omega}$ denotes the complex conjugate of $\omega$. The symmetric unitary matrix $F$ is the so-called Fourier matrix [11, p.31]. From (2.2) and (2.3) we get

(2.4) $C=F^{*} \Lambda F, \quad \Lambda=\operatorname{diag}\left(\lambda_{0}, \ldots, \lambda_{m-1}\right)=\sum_{j=0}^{m-1} c_{j} \Omega^{j}$, 
i.e. the eigenvalues $\lambda_{k}$ of $C$ are the values of the polynomial $p(z):=$ $\sum_{j=0}^{m-1} c_{j} z^{j}$ in the points $\omega^{k}, k=0, \ldots, m-1$. The vector $l$ with elements $\lambda_{0}, \ldots, \lambda_{m-1}$ is thus given by

$$
l=p(\Omega)=\sqrt{m} F^{*} c .
$$

Therefore, computing the eigenvalues of a circulant matrix merely amounts in performing an inverse Fourier transform (harmonic synthesis), which can, if $m$ is a power of 2 , be done by the fast Fourier transform in $O(m \log m)$ flops. A flop is the computational cost for a floating point add and a floating point multiply [16, p.32].

Let us now assume that $C$ is real symmetric, i.e. that $c \in \mathbf{R}^{m}$ and $c_{k}=c_{m-k}$ for $k=1, \ldots, m-1$. Then the eigenvalues of $C$ are real and it is easy to verify that

$$
\lambda_{k}=\lambda_{m-k}, \quad k=1, \ldots, m-1 .
$$

At most $\lambda_{0}$ and $\lambda_{\frac{m}{2}}$ for even $m$ can be simple eigenvalues! The eigenvectors $F^{*} e_{k}$ and $F^{*} e_{m-k}$ corresponding to $\lambda_{k}$ are elementwise complex conjugate such that their real and imaginary parts form real orthogonal eigenvectors for $\lambda_{k}$. From (2.5) one easily deduces a real representation for $\lambda_{k}$ and even $m$,

$$
\lambda_{k}=c_{0}+2 \sum_{j=1}^{m / 2-1} c_{j} \cos 2 \pi k j / m+(-1)^{k} c_{m / 2} .
$$

A similar expression holds for odd $m$.

3. Restricted eigenvalue problems. In this section we recall the theory of restricted eigenvalue problems of the form (1.4)

$$
P A P x=\lambda x, \quad P x=x,
$$

as it was treated in [1]. Here $A \in \mathbb{R}^{n \times n}$ is a symmetric matrix and $P \in \mathbb{R}^{n \times n}$ is an orthogonal projector. Let $V \in \mathbb{R}^{n \times r}$ be a matrix with orthonormal columns spanning $\mathcal{R}(P)^{\perp}$. Then $P=I-V V^{*}$ and (3.1) is equivalent to

$$
(A-\lambda I) x=V a, \quad V^{*} x=0,
$$


for some $a \in \mathbb{R}^{r}$. So $\lambda$ is an eigenvalue of (3.1) if and only if the matrix

$$
\left(\begin{array}{cc}
\lambda I-A & V \\
V^{*} & 0
\end{array}\right) \in \mathbb{R}^{m \times m}, \quad m=n+r,
$$

is singular.

Provided $\lambda$ is not an eigenvalue of $A$, the block $L D L^{T}$ decomposition [16, p.84] of the $2 \times 2$ block matrix in (3.3) yields [1]

$$
\begin{aligned}
\operatorname{det} Z(\lambda) & :=\operatorname{det} V^{*}(A-\lambda I)^{-1} V \\
& =\operatorname{det}\left(\begin{array}{cc}
\lambda I-A & V \\
V^{*} & 0
\end{array}\right) / \operatorname{det}(A-\lambda I) .
\end{aligned}
$$

Therefore, the eigenvalues of the restricted eigenvalue problem (3.1) which are not at the same time eigenvalues of $A$ can be determined by finding the roots of the equation

$$
\operatorname{det} Z(\lambda)=0 .
$$

Often, (3.5) is referred to as secular equation $[15,16]$. Mainly in the literature on the methods of intermediate problems $\operatorname{det} V^{*}(A-$ $\lambda I)^{-1} V$ is called Weinstein determinant $[23,24]$. In structural engineering this determinant is known as Kron's determinant [20].

If $\operatorname{det} Z(\lambda)$ vanishes, the eigenspace of (3.1) corresponding to $\lambda$ can be constructed from the nullspace of $Z(\lambda)$. Furthermore, not only the determinant but also the inertia of $Z(\lambda)$ gives information about the location of the eigenvalues of (3.1). We resume the results in the following Theorem, which is valid for any $\lambda \in \mathbb{R}$.

THEOREM 3.1. [1] Let $\lambda \in \mathbf{R}$. Let $\mu \geq 0$ be the multiplicity of $\lambda$ as eigenvalue of $A$ and let $W \in \mathbb{R}^{n \times \mu}$ be a matrix the columns of which form an orthonormal basis of the eigenspace $\mathcal{N}(A-\lambda I)$ corresponding to $\lambda$. Let $\nu(\lambda)$ be the number of negative eigenvalues of the extended Weinstein matrix

(3.6) $\quad Z_{+}(\lambda):=\left(\begin{array}{cc}V^{*}(A-\lambda I)^{+} V & V^{*} W \\ W^{*} V & 0\end{array}\right) \in \mathbb{R}^{(r+\mu) \times(r+\mu)}$.

If $N(\lambda)$ and $\tilde{N}(\lambda)$ denote the numbers of the eigenvalues of $A$ and of the restricted eigenvalue problem (3.1), respectively, which 
are $\leq \lambda$ then

$$
\tilde{N}(\lambda)+\nu(\lambda)=N(\lambda)
$$

The mapping defined by

$$
\left(\begin{array}{l}
a \\
z
\end{array}\right) \longmapsto x:=(A-\lambda I)^{+} V a+W z
$$

bijectively maps the nullspace of $Z_{+}(\lambda)$ onto the eigenspace of the restricted eigenvalue problem corresponding to $\lambda$.

$Z_{+}(\lambda)$ is a generalization of $Z(\lambda)$ : If $\lambda$ is in the resolvent set of $A$, we have $\mu=0$ and the Moore-Penrose generalized inverse $(A-\lambda I)^{+}$of $A-\lambda I$ coincides with $(A-\lambda I)^{-1}$. In this case (3.8) takes on the simple form

$$
a \longmapsto x:=(A-\lambda I)^{-1} V a .
$$

The extended Weinstein matrix is the limit of the matrix

$$
\left(\begin{array}{cc}
V^{*} Q^{\perp}(A-\xi I)^{-1} Q^{\perp} V & V^{*} W \\
W^{*} V & (\xi-\lambda) I_{\mu}
\end{array}\right), \quad \xi \notin \sigma(A),
$$

for $\xi$ tending to $\lambda$. Here $Q^{\perp}=I-W W^{*}$ is the orthogonal projector on $\mathcal{R}(W)^{\perp}=\mathcal{N}(A-\lambda I)^{\perp}$. The matrix in (3.9) is constructed in such a way that only the components of the columns of $V$ in $\mathcal{R}(W)^{\perp}$ are multiplied by $(A-\xi I)^{-1}$ while the components in $\mathcal{R}(W)$ remain essentially unchanged. In the unmodified Weinstein matrix this components are multiplied by $(\xi-\lambda)^{-1}$ which causes a pole in the Weinstein determinant at $\lambda$. So it is not surprising that the matrix (3.9) is congruent with

$$
V^{*}(A-\xi I)^{-1} V \oplus(\xi-\lambda) I_{\mu}, \quad \xi \notin \sigma(A),
$$

with respect to a unit triangular matrix [1]. Its determinant is therefore bounded in a neighbourhood of the eigenvalue $\lambda$ and the relation

$$
\lim _{\xi \rightarrow \lambda}(\xi-\lambda)^{\mu} \operatorname{det} Z(\xi)=\operatorname{det} Z_{+}(\lambda)
$$


holds. In a similar way more than one pole can be smoothed out from the Weinstein determinant [1].

Denoting the nondecreasingly ordered eigenvalues of $A$ and of (3.1), respectively, by $\lambda_{0}, \ldots, \lambda_{m-1}$ and $\tilde{\lambda}_{0}, \ldots, \tilde{\lambda}_{n-1}$, we can easily get from (3.7) the interlacing property

$$
\lambda_{k} \leq \tilde{\lambda}_{k} \leq \lambda_{k+r}, \quad 0 \leq k<n
$$

4. The banded symmetric Toeplitz eigenvalue problem. In this section we apply the theory of the two previous sections on the restricted eigenvalue problem (1.4)-(1.5) and formulate an algorithm for the computation of a few of its smallest eigenvalues.

The orthogonal projector $P$ in (1.3) can be written as $P=$ $I-V V^{*}$ with

$$
V:=\left(\begin{array}{c}
I_{r} \\
0
\end{array}\right) \in \mathbf{R}^{m \times r}
$$

With the easily obtainable spectral decomposition (2.4) of $C$, the Weinstein matrix in (3.6) becomes

(4.1) $\left(\begin{array}{cc}V^{*}(C-\lambda I)^{+} V & V^{*} W \\ W^{*} V & 0\end{array}\right)=\left(\begin{array}{cc}U^{*}(\Lambda-\lambda I)^{+} U & V^{*} W \\ W^{*} V & 0\end{array}\right)$.

The matrix $U^{*}:=(F V)^{*} \in \mathbf{R}^{r \times m}$ consists of the first $r$ components of the eigenvectors of $C$ while $V^{*} W \in \mathbb{R}^{r \times \mu}$ consists of the first $r$ components of the eigenvectors corresponding to $\lambda$. The elements of the submatrices of the Weinstein matrix are given by

$(4.2)\left(U^{*}(\Lambda-\lambda I)^{+} U\right)_{i j}=\frac{1}{m} \sum_{\substack{k=0 \\ \lambda_{k} \neq \lambda}}^{m-1} \frac{\omega^{k(i-j)}}{\lambda_{k}-\lambda}, \quad 0 \leq i, j \leq r-1$,

and by

$$
\left(V^{*} W\right)_{i h}=g_{\eta i}, \quad 0 \leq i \leq r-1, \quad 1 \leq h \leq \mu .
$$


Here, $h \mapsto \eta$ is a bijective map from $\{1, \ldots, \mu\}$ on $\left\{k \mid \lambda_{k}=\lambda\right\}$. As in (2.7) we may write for even $m$ and $\lambda \notin \sigma(C)$

$$
\begin{aligned}
& \left(U^{*}(\Lambda-\lambda I)^{-1} U\right)_{i j}=\frac{1}{m}\left[\frac{1}{\lambda-\lambda_{0}}\right. \\
& \left.\quad+2 \sum_{j=1}^{\frac{m}{2}-1} \frac{1}{\lambda-\lambda_{j}} \cos \frac{2 \pi k(i-j)}{m}+\frac{(-1)^{k}}{\lambda-\lambda \frac{m}{2}}\right] .
\end{aligned}
$$

A similar equation holds for odd $m$. If $\lambda \in \sigma(C)$ the corresponding expression for $\left(U^{*}(\Lambda-\lambda I)^{+} U\right)_{i j}$ is obtained from (4.4) by omitting the terms with vanishing denominator. Thus $\left(U^{*}(\Lambda-\lambda I)^{+} U\right)$ is a real symmetric Toeplitz matrix! If we assume that the values $2 / m \cos 2 \pi k l / m, 0 \leq k<m, 0 \leq l<r$, are precomputed, the cost of the calculation of $Z(\lambda) \in \mathbf{R}^{r \bar{x} r}$ is only $r m / 2+O(m+r)$ flops. (The assumption is reasonable as these values are already needed when computing the eigenvalues $\lambda_{k}$ by (2.7).

Let $\hat{\lambda}_{0} \leq \cdots \leq \hat{\lambda}_{m-1}$ be the eigenvalues of $C$ arranged in nondecreasing order. Then, because of (3.9), to compute the $s$ smallest eigenvalues $\tilde{\lambda}_{0}, \ldots, \tilde{\lambda}_{s-1}$ of $T$ we can restrict ourselves to the investigation of the Weinstein matrix in the interval $\left[\hat{\lambda}_{0}, \hat{\lambda}_{s+r-1}\right]$. By the inertia of the generalized Weinstein matrix evaluated for the eigenvalues $\hat{\lambda}_{0}, \ldots, \hat{\lambda}_{s+r-1}$ we can determine, which of these eigenvalues persist, i.e. are eigenvalues of $T$ as well as of $C$. (Note that at most two of these eigenvalues are simple.) Corresponding eigenvectors are obtained by (3.8). At the same time, using (3.7) we can determine the number of eigenvalues of $T$ in each open subinterval $\left(\hat{\lambda}_{k}, \hat{\lambda}_{k+1}\right)$. Then in each subinterval which contains at least one eigenvalue of $T$, the zeros of the secular equation (3.5) can be found by some rootfinder.

These considerations immediately lead to the following

Algorithm 4.1. Let $T \in \mathbb{R}^{n \times n}$ be a symmetric banded Toeplitz matrix with bandwidth $r$. This algorithm computes the $s$ smallest eigenvalues of $T$.

1. (a) Compute all eigenvalues $\lambda_{i}, 0 \leq i \leq m-1, m=n+r$, of the circulant matric $C$ as defined in (1.5) and the first $r$ components of the corresponding eigenvectors.

(b) Sort the eigenvalues $\lambda_{i}$ into $\hat{\lambda}_{i}$ such that $\hat{\lambda}_{i} \leq \hat{\lambda}_{i+1}$. Determine $m^{\prime} \in \mathbb{Z}$ and the map $\sigma: \mathbb{Z} \supset\left[0, \bar{m}^{\prime}-1\right]$ 


$$
\begin{aligned}
& \longrightarrow[0, m-1] \subset \mathbb{Z}, m^{\prime} \leq m, \text { such that } \\
& \bigcup_{0 \leq j \leq m^{\prime}-1}\left\{\hat{\lambda}_{\sigma(i)}\right\}=\bigcup_{0 \leq j \leq m-1}\left\{\hat{\lambda}_{j}\right\}
\end{aligned}
$$

and

$$
\hat{\lambda}_{\sigma(i)}<\hat{\lambda}_{\sigma(i+1)}, 0 \leq i<m^{\prime}-1
$$

(c) Determine $s^{\prime}$ such that $\hat{\lambda}_{\sigma\left(s^{\prime}\right)}=\lambda_{r+s-1}$.

2. Compute the extended Weinstein matrix

$$
Z_{+}(\lambda)=\left(\begin{array}{cc}
U^{*}(\Lambda-\lambda I)^{+} U & V^{*} W \\
W^{*} V & 0
\end{array}\right)
$$

for $\lambda=\hat{\lambda}_{\sigma(1)}, \ldots, \hat{\lambda}_{\sigma\left(s^{\prime}\right)}$ and determine its inertia. The zero inertia of $Z_{+}\left(\hat{\lambda}_{\sigma(j)}\right)$ equals the multiplicity of $\hat{\lambda}_{\sigma(j)}$ as eigenvalue of $T$.

3. Determine the number of the eigenvalues in the intervals $\left(\hat{\lambda}_{\sigma(j)}, \hat{\lambda}_{\sigma(j+1)}\right), 0 \leq j<s^{\prime}$, and compute them (if any) by finding the zeros of the secular equation.

Clearly, the $s^{\prime}+1$ tasks in step 2 are mutually independent. They can be performed in parallel as soon as the preparatory step 1 has been completed.

That task in step 3 computing the zeros of the Weinstein determinant in the interval $\left(\hat{\lambda}_{\sigma(j)}, \hat{\lambda}_{\sigma(j+1)}\right)$ can be started as soon as the two tasks of step 2 corresponding to $\hat{\lambda}_{\sigma(j)}$ and $\hat{\lambda}_{\sigma(j+1)}$ have been finished. All $s^{\prime}$ tasks in step 3 are mutually independent. Thus we get a data dependancy graph for the above algorithm for the case $s^{\prime}=4$ as depicted in Fig. 1 . Node 1 corresponds to step 1 of the algorithm. Nodes 2 to 6 correspond to the $s^{\prime}+1$ tasks of step 2 while nodes 7 to 10 correspond to step 3 . The sole purpose of node 11 is to check if all tasks of step 3 have completed. This node is needed by Schedule [14] which was used to implement our algorithm an Alliant FX-8.

The amount of work in node 1 is $\frac{7}{2}(r+1) m+O(r)$ flops. This includes the computation of the eigenvalues of $C$ and the constants $\frac{2}{m} \cos 2 \pi k l / m$ but not the sorting of the eigenvalues. The 


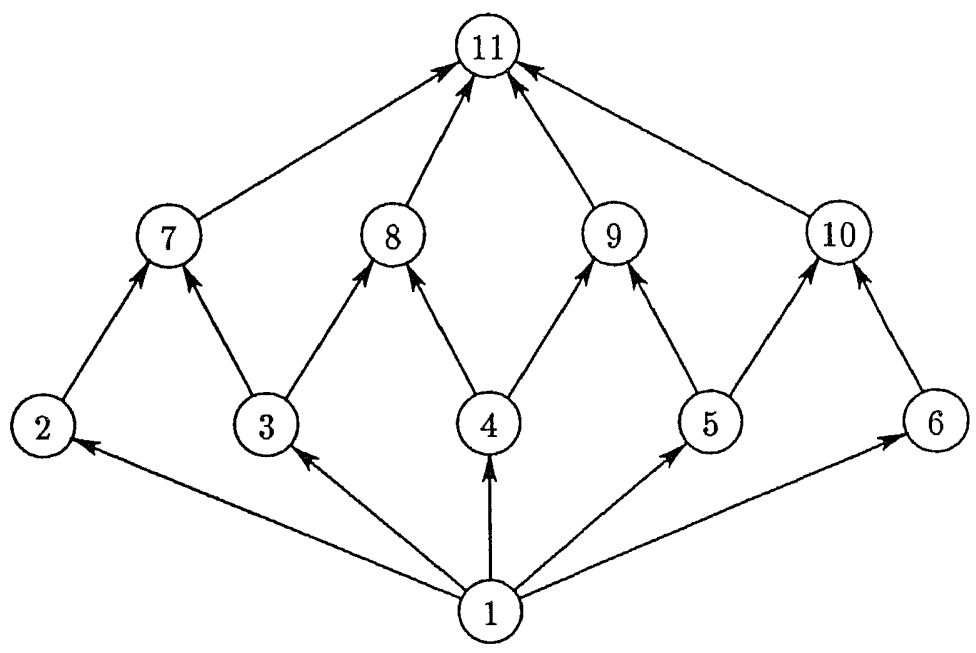

FIG. 1. Data dependency graph with $s^{\prime}=4$.

work to be done in each of the nodes of step 2 is $r m / 2+(r+$ $\mu)^{3} / 6+O(r+\mu+m)$ flops. $\mu$ will usually be 2 . The amount of work in each of the nodes corresponding to step 3 is essentially the number of Weinstein matrix evaluations times $r m / 2+r^{3} / 6$. If the zeros of the Weinstein determinant were to be computed by bisection this number would be fairly high. The number of bisection steps to shrink a bracketing interval of length $l$ to one of length $\leq \epsilon l$ is $\left\lceil-\log _{2} \epsilon\right\rceil$. For $\epsilon=10^{-15}$ this number is 50. So the amount of work to compute one eigenvalue of $T$ to machine precision is around $50\left(\mathrm{rm} / 2+r^{3} / 6\right)$. The sequential complexity of the computation of $s$ eigenvalues of $T$ is thus $O\left(s r\left(m+r^{2}\right)\right)$.

Remark 1. If $r$ is not small and if $m$ is a power of 2 , the Weinstein matrix can be computed by the fast Fourier transform in $O\left(m \log _{2} m\right)$ flops.

Remark 2. An eigenvector corresponding to an eigenvalue $\tilde{\lambda} \notin$ 
$\sigma(C)$ is obtained by

$\tilde{x}=(C-\tilde{\lambda} I)^{-1} V a=F^{*}(\Lambda-\tilde{\lambda} I)^{-1} F V a, \quad a \in \mathcal{N}(Z(\tilde{\lambda})) \subset \mathbb{R}^{r}$.

The computation of $\tilde{x}$ costs $O\left(m \log _{2} m+r m\right)$ flops if the fast Fourier transform can be applied.

Remark 3. The modification of the above theory to the generalized eigenvalue problem $T x=\lambda S x, T, S$ Toeplitz and $S$ positive definite, is straightforward if $r$ is defined to be the maximum of the bandwidths of $T$ and $S$ and if the circulant matrix corresponding to $S$ is still positive definite.

5. Numerical experiments. In this section we discuss three implementations of Algorithm 4.1. They differ from each other only in the way the zeros of the Weinstein determinant are computed in step 3 .

In the first implementation each task of step 3 is executed on a single processor. The zeros of the Weinstein determinant are determined by a bisection algorithm analogous to the one proposed by Barth et al. [3] This implementation works solely with the inertia of the Weinstein matrix. The inertia are computed by the Bunch-Kaufman algorithm [9] as implemented in the LINPACK routines dsifa and dsidi [12].

The second implementation is a refinement of the first one. If a point in an interval is found dividing the set of eigenvalues of the interval in two, a process is spawned dynamically to compute the eigenvalues below the separation point. The spawning process continues work on the eigenvalues above this point. The idea to parallelize bisection this way has been proposed by Bernstein and Goldstein [5].

The third implementation is a further refinement of the second one: As soon as an interval containing only one zero is detected, bisection is replaced by the zerofinder of Brent [8] which is available as ForTRAN procedure zeroin from netlib [13]. zeroin is applied on the bounded function $f(\xi)=\left(\xi-\hat{\lambda}_{\sigma(k)}\right)^{\mu_{\sigma(k)}}(\xi-$

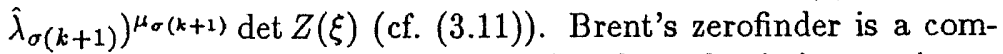
bination of bisection, secant method and quadratic inverse interpolation. Its convergence rate is $\approx 1.618$. As only one func- 
tion evaluation per iteration step is required the method has an efficiency index $\approx 1.618$, which is higher than the efficiency in$\operatorname{dex} \sqrt{2} \approx 1.414$ of Newton's method.

These algorithms are compared with the combination of EIsPACK routines which computes a few of the smallest eigenvalues of (general) symmetric banded matrices [21]. Subroutine bandr reduces a banded matrix to tridiagonal form while subroutine tridib computes selected eigenvalues of the tridiagonal matrix by the bisection algorithm mentioned above.

TABLE 1

Timings for the different implementations in seconds

\begin{tabular}{|c|c|c|c|c|c|c|c|c|}
\hline$n$ & $r$ & $s$ & $s^{\prime}$ & $\mathrm{p}$ & $\begin{array}{c}\text { static } \\
\text { bisection }\end{array}$ & $\begin{array}{c}\text { dynamic } \\
\text { bisection }\end{array}$ & $\begin{array}{c}\text { dynamic } \\
\text { bisection/ } \\
\text { zeroin }\end{array}$ & $\begin{array}{c}\text { bandr/ } \\
\text { tridib }\end{array}$ \\
\hline 100 & 3 & 10 & 7 & 1 & .304 & .335 & .108 & .557 \\
& & & & 2 & .189 & .180 & .067 & \\
& & & & 3 & .160 & .145 & .055 & \\
\hline 100 & \multirow{3}{*}{25} & \multirow{2}{*}{14} & 1 & .685 & .771 & .243 & .900 \\
& & & & 2 & .398 & .410 & .140 & \\
& & & & 3 & .294 & .298 & .105 & \\
& & & & 4 & .245 & .238 & .086 & \\
& 9 & 25 & 17 & 1 & 3.66 & 3.96 & 1.18 & 7.90 \\
& & & 2 & 2.05 & 2.08 & .683 & \\
& & & & 3 & 1.42 & 1.49 & .520 & \\
\hline 300 & 9 & 50 & 30 & 1 & 7.02 & 7.62 & 2.13 & 9.30 \\
& & & & 2 & 3.70 & 3.96 & 1.16 & \\
& & & & 3 & 2.59 & 2.75 & .854 & \\
& & & & 4 & 2.18 & 2.15 & .692 & \\
\hline
\end{tabular}

In Table 1 the cpu times are listed that we obtained when the four algorithms were applied on four eigenvalue problems. We call the three implementations in the order of their introduction static bisection, dynamic bisection and dynamic bisec- 
tion/zeroin. The elements of the test Toeplitz matrices (1.1) were defined by $t_{i}:=\max \{r+1-i, 0\} . s^{\prime}$ is the number defined in part 1.(c) of Algorithm 4.1. $p$ is the number of processors used. Note that $\hat{\lambda}_{\sigma\left(s^{\prime}\right)}$ is not necessarily the smallest eigenvalue of $C$ greater or equal to $\tilde{\lambda}_{s-1}$. E.g. in the first example the sixth interval $\left(\hat{\lambda}_{5}, \hat{\lambda}_{6}\right)$ containes the single eigenvalue $\tilde{\lambda}_{10}$ of $T$. This is known after the computation of the inertia of $Z_{+}\left(\hat{\lambda}_{5}\right)$. In order not to destroy the parallelism of the algorithm all $s^{\prime}$ tasks of step 1 have to be executed. Intervals containing no eigenvalues of interest are then not investigated any further, i.e. the corresponding task is empty.

It is surprising that dynamic bisection is slower than static bisection in most cases. Of course dynamic bisection must be slower than static bisection due to the increased overhead if only one processor is used - if dynamic spawning occurs at all. But this is indeed the case as can be seen in Table 2 where the number of intervals $\left(\hat{\lambda}_{\sigma(k)}, \hat{\lambda}_{\sigma(k+1)}\right)$ is listed containing a certain number of eigenvalues $\tilde{\lambda}_{j}$ of $T$.

TABLE 2

Number of intervals containing a certain number of eigenvalues of $T$.

\begin{tabular}{cccccccc}
\hline example & 0 & 1 & 2 & 3 & 4 & 5 & 6 \\
\hline 1 & - & 2 & 1 & 2 & - & - & - \\
2 & - & 5 & 4 & 4 & - & - & - \\
3 & 4 & 2 & 5 & - & 1 & 2 & - \\
4 & 6 & 6 & 8 & 2 & 2 & 2 & 1 \\
\hline
\end{tabular}

Dynamic bisection refines the granularity of the tasks and in this way increases the potential to balance the work load of the different processors. Except for the first example it seems that $s^{\prime}$ is sufficiently big and $p$ is sufficiently small to yield fairly well balanced work loads. Dynamic bisection does not bring essential advantages if the spawned process can be executed on an idle processor. The finer granularity may be the reason that the speedups from the one to the multiprocessor version are the highest for 
dynamic bisection. If we assume that the computation of each eigenvalue needs the same amount of time and is performed in one independent task, the speedup to be expected is $s /\left\lceil\frac{s}{p}\right\rceil$. The speedups observed with dynamic bisection are above $90 \%$ of these theoretical numbers.

Dynamic bisection combined with zeroin is evidently superior to the pure bisection methods by a factor of about 3 or more. The speedups observed are however not so high as for dynamic bisection. This is a consequence of Amdahl's law: the slow parts of the program, i.e. the initial bisection steps don't parallelize as well as zeroin.

The overall speedups of dynamic bisection/zeroin on four processors compared with bandr/tridib are much higher than four. They range from 10.5 in example 2 to 17.7 in example 3.

By the timings of example 3 and 4 the different behavior of the newly developed parallel algorithms and the combination of bandr and tridib becomes evident. The parallel algorithms are built up of components all with $O(\mathrm{rm})$ complexity. Thus the overall work is proportional to the number $s$ of desired eigenvalues.

The EISPACK routines behave completely different. An initial amount of work of $O\left(r n^{2}\right)$ flops is spent by routine bandr [19]. (For the examples with $n=300$ bandr takes about $6.5 \mathrm{sec}$.) Then work proportional to $s$ is done in tridib the complexity of which is $O(n)$ ! So the superiority of the parallel algorithms decreases with increasing $s$. Big $r$ 's are not disadvantageous as long as the $O\left(r^{3}\right)$ parts of the algoritm i.e. the computation of inertia/determinant of $Z(\lambda)$ does not outweight the computation of $Z(\lambda)$ itself. Big values of $r$ would have a much higher influence if the Weinstein matrix were not Toeplitz as it were the case if we considered the restricted eigenvalue problem of an arbitrary symmetric matrix $A$ whose spectral decomposition is known (cf. §3). In this more general situation numerical results would certainly not be so favorable for the parallel algorithms. 


\section{REFERENCES}

[1] P. ARbenz, W. Gander, AND G. H. Golub, Restricted rank modification of the symmetric eigenvalue problem: Theoretical considerations, Linear Algebra Appl., 104 (1988), pp. 75-95.

[2] P. ARbenz and G. H. Golub, On the spectral decomposition of Hermitian matrices modified by low rank perturbations with applications, SIAM J. Matrix Anal. Appl., 9 (1988), pp. 40-58.

[3] W. Barte, R. S. Martin, AND J. H. Werinson, Calculation of the eigenvalues of a symmetric tridiagonal matrix by the method of bisection, Numer. Math., 9 (1967), pp. 386-393. Also in: Linear Algebra, J.H. Wilkinson, C. Reinsch (eds.), pp. 249-256, Springer, Berlin, 1971.

[4] C. BEATtE AND D. Fox, Schur complements and the WeinsteinAronszajn theory for modified matrix eigenvalue problems, Tech. Rep. UMSI 87/11, University of Minnesota Supercomputer Institute, February 1987.

[5] H. J. Bernstern AND M. Goldstein, Parallel implementation of bisection for the calculation of eigenvalues of tridiagonal symmetric matrices, Computing, 37 (1986), pp. 85-91.

[6] D. BINI AND M. CAPOVANI, Spectral and computational properties of band symmetric Toeplitz matrices, Linear Algebra Appl., 53 (1983), pp. 99-126.

[7] D. BINI AND V. PAN, Efficient algorithms for the evaluation of the eigenvalues of (block) banded Toeplitz matrices, Math. Comp., 50 (1988), pp. 431-448.

[8] R. P. BRENT, Algorithms for Minimization without Derivatives, Series in Automatic Computation, Prentice-Hall, Englewood Cliffs, 1973.

[9] J. R. BUNCB AND L. KaUfman, Some stable methods for calculating inertia and solving symmetric linear systems, Math. Comp., 31 (1977), pp. 163-179.

[10] G. CYBenko AND C. VAN LOAN, Computing the minimum eigenvalue of a symmetric positive definite Toeplitz matrix, SIAM J. Sci. Statist. Comput., 7 (1981), pp. 123-131.

[11] P. J. Davis, Circulant Matrices, Wiley, New York, 1979.

[12] J. J. Dongarra, J. R. Bunch, C. B. Moler, and G. W. StewART, LINPACK Users Guide, SIAM, Philadelphia, 1978.

[13] J. J. Dongarra AND E. Grosse, Distribution of mathematical software via electronic mail, Comm. ACM, 30 (1987), pp. 403-407.

[14] J. J. Dongarra and D. C. SORENSEN, A portable environment for developing parallel FORTRAN programs, Parallel Computing, 5 (1987), pp. 175-186.

[15] G. H. GOLUB, Some modified matrix eigenvalue problems, SIAM Rev., 15 (1973), pp. 318-334.

[16] G. H. GoluB AND C. F. van Loan, Matrix Computations, The Johns Hopkins University Press, Baltimore, 1983. 
[17] Y. H. HU AND S. Y. KUNG, Toeplitz eigensystem solver, IEEE Trans. Acoustics, Speech, Signal Proc., ASSP-33 (1985), pp. 1264-1271.

[18] A. K. JaIN, Fast inversion of banded Toeplitz matrices by circular decompositions, IEEE Trans. Acoustics, Speech, Signal Proc., ASSP26 (1978), pp. 121-126.

[19] H. R. SCBWARZ, Tridiagonalization of a symmetric band matrix, Numer. Math., 12 (1968), pp. 231-241. Also in: Linear Algebra, J.H. Wilkinson, C. Reinsch (eds.), pp. 273-283, Springer, Berlin, 1971.

[20] A. SIMPson, The Kron methodology and practical algorithms for eigenvalue, sensitivity and response analyses of large scale structural systems, Aeronaut. J., 84 (1980), pp. 417-433.

[21] B. T. Smith, J. M. Boyle, J. J. Dongarra, B. S. Garbow, Y. IKEBE, V. C. KLEMA, AND C. B. MOLER, Matrix Eigensystem Routines - EISPACK Guide, Lecture Notes in Computer Science 6, Springer, Berlin, 1976.

[22] W. F. TrencB, On the eigenvalue problem for Toeplitz band matrices, Linear Algebra Appl., 64 (1985), pp. 199-214.

[23] H. F. Weinberger, Variational Methods for Eigenvalue Approximation, Regional Conference Series in Applied Mathematics 15, SIAM, Philadelphia, 1974.

[24] A. Weinstein AND W. STEnger, Methods of Intermediate Problems for Eigenvalues, Academic Press, New York, 1972. 